\title{
Involvement of Nitric Oxide in Noradrenaline-Induced Changes in the Activity of Antioxidant Enzymes and Lipid Peroxidation in Rat Brown Adipose Tissue and Heart
}

\author{
A. PEROVIC 1 , T. VUCKOVIC ${ }^{2}$, G. CVIJIC ${ }^{2}$, J. DJORDJEVIC ${ }^{2}$, V. DAVIDOVIC ${ }^{2}$ \\ ${ }^{1}$ Faculty of Biology, University of Podgorica, Republic of Montenegro, ${ }^{2}$ Institute of Physiology and \\ Biochemistry, Faculty of Biology, University of Belgrade, Republic of Serbia
}

Received May 16, 2006

Accepted December 27, 2006

On-line available January 2, 2007

\begin{abstract}
Summary
The effect of exogenous noradrenaline (NA) (1.6 mg. $\mathrm{kg}^{-1}$ i.p., $35 \mathrm{~min}$ prior sacrifice) on the activity of antioxidant enzymes (AOE) copper zinc superoxide dismutase (CuZnSOD), manganese superoxide dismutase (MnSOD) and catalase (CAT), as well as lipid peroxides (LP) concentration were studied in the rat interscapular brown adipose tissue (IBAT) and heart of saline (controls) and $\mathrm{N}^{\omega}$-nitro-L-arginine methyl ester (L-NAME) treated rats ( $10 \mathrm{mg} . \mathrm{kg}^{-1}$, i.p., during 3 days and $20 \mathrm{~min}$ before NA). NA differently affects both $A O E$ activities and LP production in the IBAT and heart. Thus, NA inhibited the activity of all IBAT AOE and LP production while in the heart it markedly increased CAT activity only, but had no effect on any of SODs activities and LP concentration. L-NAME, a nitric oxide synthase blocker, completely abolished the NA-induced inhibition of the IBAT AOE and LP production, whereas in the heart it was without effect. In conclusion, these results indicate that both NA and L-NAME effects on AOE activity and LP production are tissue specific and also suggest that nitric oxide mediates the NA-induced inhibition of AOE activity and LP production in the IBAT only.
\end{abstract}

\section{Key words}

Antioxidant enzymes • Brown adipose tissue • Heart • L-NAME • Noradrenaline

\section{Corresponding author}

Dr Vukosava Davidovic, Institute of Physiology and Biochemistry, Faculty of Biology, University of Belgrade. Studentski Trg 16, PO BOX 52, 11000 Belgrade, Republic of Serbia, E-mail: vukana@bf.bio.bg.ac.yu

\section{Introduction}

It is well known that noradrenaline (NA) exerts widespread effects in many body tissues including the brown adipose tissue (BAT) and heart. The BAT functions as a metabolic buffer (Himms-Hagen 1990) in all situations when energy balance is changed. Its thermogenic activity is under neural and hormonal control. Noradrenaline, released from sympathetic nerves, directly controls the uncoupling protein-1 synthesis (Ricquer and Cassard-Doulcier 1993). This mitochondrial protein represents the molecular marker of the BAT metabolic activity. However, it has been shown that NA can mediate the vasodilatation by stimulating nitric oxide (NO) production in the BAT, resulting in an increase in the BAT blood flow (Nagashima et al. 1994). It appears that NO is also involved in the enhancement of the thermogenic function of BAT in rats (Saha and Kuroshima 2000). There are data suggesting that endothelial nitric oxide synthase (eNOS) is primary isoform that is responsible for NO production in the BAT and that its expression may be under sympathetic control (Kikuchi-Utsumi et al. 2002). However, Nisoli et al. (1997) found that NO, generated by the stimulation of inducible NOS (iNOS) in brown adipocytes, may represent an important mechanism in the modulation of varying BAT functions among which is vasodilatation of the BAT blood vessels. At the same time, Koivisto et al. (1997) showed that NO inhibits the respiration in mitochondria isolated from the rat brown adipose tissue. Finally, there is evidence suggesting that brown adipocytes contain noradrenergically modulated nuclear enzyme systems capable to produce biologically active gaseous mediator such as NO, which can cooperate in controlling brown fat cell biology (Giordano et al. 2002). Nevertheless, there are data that antioxidant enzymes may be inactivated by peroxynitrite $\left(\mathrm{ONOO}^{-}\right)$. The latter 
molecule is responsible for the inactivation of a specific tyrosine residue in MnSOD and some mitochondrial proteins (Yamakura et al. 1998). Therefore, when mitochondria are exposed to NO, NO diffuses through the membranes and reversibly inhibits cytochrome oxidase. This inhibition causes the inhibition of the mitochondrial respiratory chain and as a consequence increases mitochondrial superoxide anion release that reacts with NO, leading to peroxynitrite formation (Cassina and Radi 1996). Peroxynitrite then irreversibly inhibits complexes I and II in the mitochondrial respiratory chain. In this mechanism, the accumulation of superoxide anion results in the production of a large amount of peroxynitrite. Namely, superoxide, known as NO scavengers, drastically reduces NO bioactivity (Napoli and Ignarro 2001).

In addition to their thermogenic and vasodilatatory effects, noradrenaline has the antioxidant action (Sergeev et al. 1975) that was associated with the presence of a free phenol group in its molecules. The mechanism of inhibition is the interaction of noradrenaline with the free radicals leading to antioxidative protection. Furthermore, catecholamines inhibit lipid peroxidation (Andorn and Pappola 2001). It is also well known that the rise in NO concentration may affect the activity of some antioxidant enzymes, leading to an imbalance of the prooxidant versus antioxidant status and an increase in the production of reactive oxygen species. Thus, it was shown that NO donors, Snitroso-N-acetylpenicillamine (SNAP) and sodium nitropruside, inhibit catalase activity in a concentrationdependent manner (Kremser et al. 1995) and that LNAME, an NO synthase inhibitor, abolishes a decrease in the activities of antioxidative enzymes in immobilizationstressed rats, i.e. under the conditions when the activity of sympathetic nervous system and noradrenaline release are increased (Kostic et al. 2000).

Pharmacological and molecular studies also suggested the existence of a constitutive NOS (cNOS) in cardiac myocytes (Finkel et al. 1992, Kanai et al. 1997) and that noradrenaline and adrenaline increase myocardial contractility by elevating cytoplasmic $\mathrm{Ca}^{2+}$ levels, which also stimulate NO release and may provide a mechanism for the coronary artery vasodilatation and enhanced diastolic relaxation observed with adrenergic stimulation.

Bearing in mind the facts mentioned above, we examined whether NO regulates the activities of antioxidant enzymes in IBAT and heart as well as peroxides concentration in rats treated with exogenous NA. This has been carried out by determining the effects of L-NAME, which is an NOS inhibitor, and NA on the rat oxidative status in the IBAT and heart.

\section{Methods}

\section{Animals and treatment}

Male rats of the Wistar strain (Rattus norvegicus), three months old, were used for the experiments. All experiments were conducted in accordance with the rules of animals care proposed by the Serbian Association of Laboratory Animal Science (SALAS). The animals acclimated to $22 \pm 1{ }^{\circ} \mathrm{C}$ and maintained under intermittent 12 -h periods of light and dark, had free access to commercial rat food (Subotica, Serbia and Montenegro) and tap water.

The rats were divided into two main groups, each consisting of 12 animals. The first group was divided into two subgroups. The first subgroup, controls (vehicle) was treated with saline only. The second subgroup was treated intraperitoneally with noradrenaline (Sigma, St Louis, USA) in a dose of $1.6 \mathrm{mg} / \mathrm{kg} 35 \mathrm{~min}$ before sacrifice. The second group of rats was also divided into two subgroups. The first subgroup was treated intraperitoneally with L-NAME (Sigma, St Louis, USA) in dose of $10 \mathrm{mg} / \mathrm{kg}$ during four days. The last dose was given $55 \mathrm{~min}$ before animals were killed by decapitation with a guillotine (Harvard-Apparatus, Holliston, MA, USA). The second subgroup was treated intraperitoneally with L-NAME during four days and with noradrenaline $35 \mathrm{~min}$ before sacrifice. IBAT and heart were then rapidly excised, dissected, weighed and stored at $-20{ }^{\circ} \mathrm{C}$ prior to enzyme activity and lipid peroxide concentration measurements.

\section{Antioxidant enzymes assays in the IBAT and heart}

The IBAT or heart were homogenized in a buffer, containing $0.25 \mathrm{M}$ sucrose, $0.05 \mathrm{M}$ TRIS and $1 \mathrm{nM}$ EDTA, $\mathrm{pH}=7.4$, sonicated (three times at $100 \mathrm{~W}$ for $20 \mathrm{~s}$ with $10 \mathrm{~s}$ pause in a Bronson model B-12 sonicator), as described by Takada et al. (1982) to release MnSOD. The samples were then centrifuged at $20000 \mathrm{rpm}$ in a Sorvall centrifuge for $120 \mathrm{~min}$ and used for the determination of CAT, CuZnSOD and MnSOD activities. SOD activity was determined by the adrenaline method of Misra and Fridovich (1972) based on the spectrophotometrical measurement of the degree of adrenaline autooxidation inhibition by SOD contained in the 


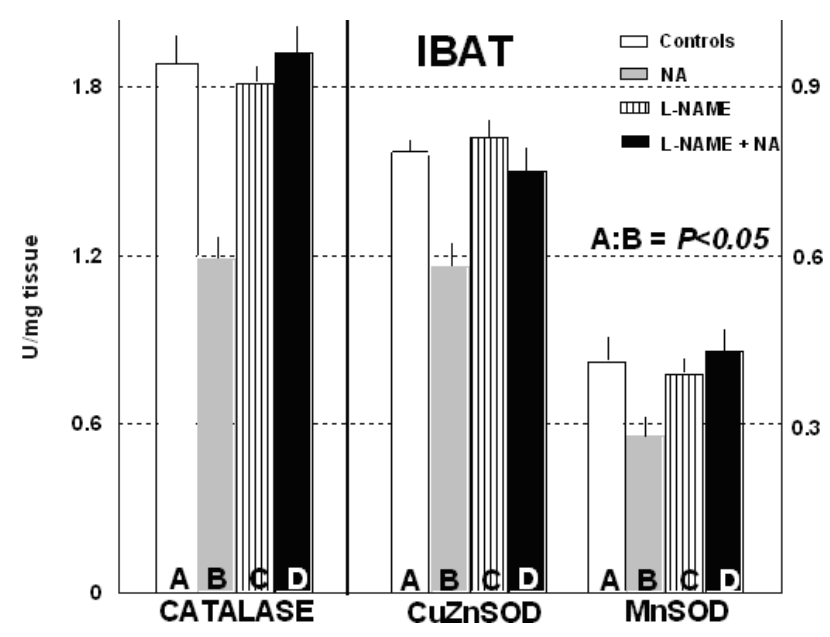

Fig. 1. Effect of noradrenaline $\left(1.6 \mathrm{mg}\right.$. $\mathrm{kg}^{-1}$ i.p., $35 \mathrm{~min}$ prior sacrifice) and L-NAME (10 mg. $\mathrm{kg}^{-1}$, i.p., during 3 days and 20 min before noradrenaline) on the IBAT antioxidant enzymes activity (Catalase, CuZnSOD, MnSOD). Values are expressed as units of SOD or CAT/mg wet tissue weight and represent the means \pm S.E.M. of six animals.

examined samples. Total specific SOD activity and that of MnSOD (after CuZnSOD inhibition with $\mathrm{KCN}$ ) was measured, and then the CuZnSOD activity was calculated.

CAT activity was measured spectrophotometrically by the method of Beutler (1982). This method is based on the rate of $\mathrm{H}_{2} \mathrm{O}_{2}$ degradation by the action of CAT contained in the examined samples.

\section{Lipid peroxidation measurement}

The extent of the peroxidative reactions in the IBAT and heart was determined by measuring malonyl dialdehyde (MDA) concentration in the IBAT or heart homogenates (prepared in $50 \mathrm{nM}$ Tris- $\mathrm{HCl}$ buffer, $\mathrm{pH}=7.4$ in a ratio of 1:50) by the modified thiobarbituric acid method without stimulation of peroxidative processes with $\mathrm{Fe}^{2+}$ and ascorbate (Rehncrona et al. 1980).

\section{Statistics}

Student's $t$ test was used for statistical analysis of results with $\mathrm{p}<0.05$ or higher as significant difference.

\section{Results}

\section{Effects of NA and L-NAME on the IBAT and heart antioxidant enzymes activities}

Figure 1 illustrates the changes in the activity of antioxidant enzymes (CAT, CuZnSOD and MnSOD) in the IBAT of rats treated with saline (controls), noradrenaline and L-NAME. Exogenous noradrenaline

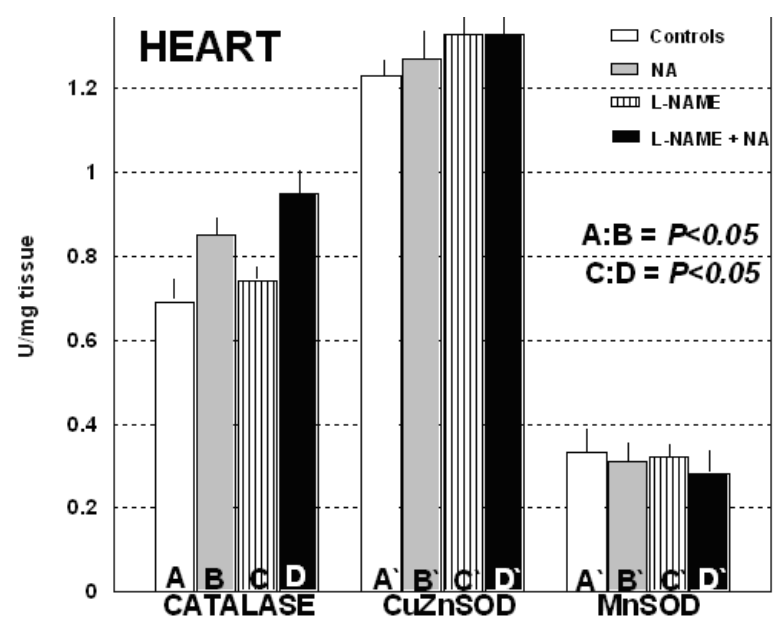

Fig. 2. Effect of noradrenaline $\left(1.6 \mathrm{mg} . \mathrm{kg}^{-1}\right.$ i.p., $35 \mathrm{~min}$ prior sacrifice) and L-NAME (10 mg. $\mathrm{kg}^{-1}$, i.p., during 3 days and $20 \mathrm{~min}$ before noradrenaline) on the heart antioxidant enzymes activity (Catalase, CuZnSOD, MnSOD). Values are expressed as units of SOD or CAT/mg wet tissue weight and represent the means \pm S.E.M. of six animals.

injected 35 min prior to sacrifice significantly decreased the activity of all antioxidative enzymes studied. L-NAME alone did not change the activity of any of the IBAT antioxidative enzymes studied. However, L-NAME, an NO synthase blocker, completely abolished the inhibition induced by noradrenaline of any of the IBAT antioxidant enzymes.

Figure 2 shows the alterations in the activity of antioxidant enzymes (CAT, CuZnSOD and MnSOD) in the heart of rats treated with saline (controls), noradrenaline and L-NAME. Unlike the IBAT, exogenous noradrenaline markedly increased catalase activity in the heart $(\mathrm{p}<0.05)$, but had no effect on both SODs activities. Similarly to the IBAT, L-NAME applied alone did not influence to the activity of all three antioxidative enzymes in heart. At the same time, L-NAME did not prevent the noradrenaline-induced increase in heart catalase activity and was also without effect on the activity of both SODs.

\section{Effects of NA and L-NAME on the IBAT and heart lipid peroxide concentration}

It is clear from Figure 3 that the extent of peroxidative processes is tissue-specific. Thus, exogenous noradrenaline significantly decresed malonyl dialdehyde (MDA) concentration in the IBAT $(\mathrm{p}<0.01)$ but had no effect on MDA concentration in the heart. L-NAME alone did not change MDA level either in the IBAT or in the heart. However, L-NAME abolished noradrenalineinduced decrease of MDA concentration in the IBAT. 


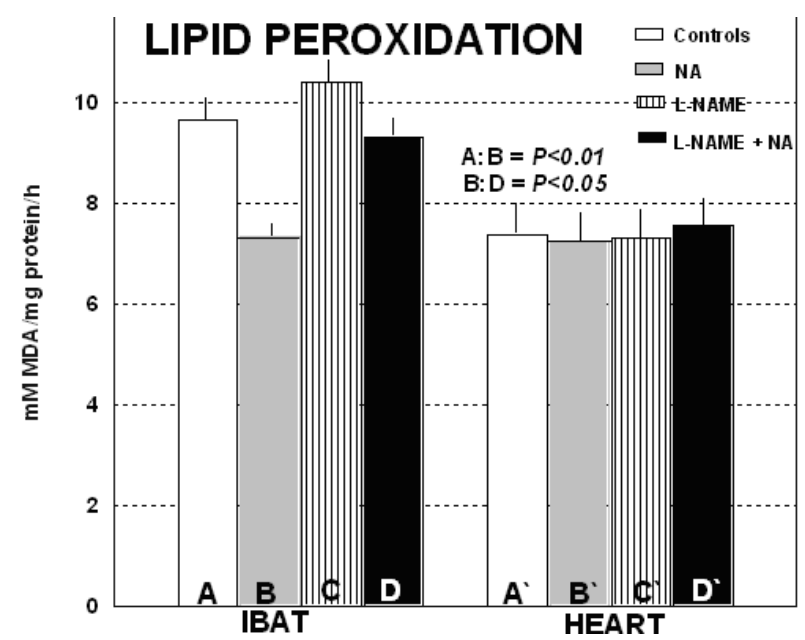

Fig. 3. Effect of noradrenaline $\left(1.6 \mathrm{mg} . \mathrm{kg}^{-1}\right.$ i.p., $35 \mathrm{~min}$ prior sacrifice) and L-NAME (10 mg. $\mathrm{kg}^{-1}$, i.p., during 3 days and $20 \mathrm{~min}$ before noradrenaline) on the MDA concentration in the IBAT and heart. MDA concentration is expressed as $\mathrm{mM} \mathrm{MDA} / \mathrm{mg}$ protein/h and represent the means \pm S.E.M. of six animals.

\section{Discussion}

Our present results clearly show that the effects of both noradrenaline and L-NAME on antioxidant enzyme activities and lipid peroxide level are tissuespecific. They also suggest that NO mediates the noradrenaline-induced inhibition of antioxidant enzyme activity and lipid peroxide concentration in the IBAT only. Thus, noradrenaline inhibited the activity of all IBAT antioxidant enzyme activities studied and lipid peroxide production, whereas in the heart it markedly increased CAT activity only, but had no effect on any of SODs activities and lipid peroxide concentration. L-NAME, a nitric oxide synthase blocker, completely abolished the noradrenaline-induced inhibition of the IBAT antioxidant enzyme activities and lipid peroxide production, but remained without effect in the heart. The observed changes are understandable if the following evidence is taken into account. First, noradrenaline can mediate the IBAT vasodilatation by stimulating the NO production leads to an increase in the IBAT blood flow (Kikuchi-Utsumi et al. 2002) and consequently the changes of their function. Second, this signaling molecule may inhibit mitochondrial respiration in isolated BAT mitochondria by a high-affinity competitive effect directly at the oxygen binding site in cytochrome c oxidase (Koivisto et al. 1997, Brown 2001). It probably also alters the production of anion free radicals affecting in this manner antioxidant enzyme activities, which leads to an imbalance of the prooxidant versus antioxidant status. Regarding the mechanism of NO action on the IBAT antioxidant enzymes, it may be supposed that this signaling molecule inhibits the activity of cytochrome oxidase in the IBAT mitochondria increasing in this way the production of superoxide radicals, which react with NO producing peroxynitrite anion (Cassina and Radi 1996). This potent biological oxidant is probably responsible for the IBAT MnSOD inactivation by tyrosine residue nitration. This is in accordance with the results of Yamakura et al. (1998) showing that the inactivation of human MnSOD by peroxynitrite is caused by nitration of tyrosine 34 to 3-nitrotyrosine. It was also suggested that NO donors inhibit CAT activity in a concentration-dependent manner (Kremser et al. 1995) and that L-NAME, an NO synthase inhibitor, abolishes a decrease in the activity of antioxidant enzymes in immobilization-stressed rats in which the activity of sympathetic nervous system and release of noradrenaline are increased (Kostic et al. 2000). It should be noted that our present data are related to the IBAT of the adults rats acclimated at room temperature of $22 \pm 1{ }^{\circ} \mathrm{C}$, when this tissue is not fully active in comparison to brown fat of cold-adapted animals. However, we have previously shown that changes in the activity of antioxidative enzymes are tissue-specific and dependent on duration of cold exposure, which is related to the role of examined tissues in the different phases of cold exposure (Davidovic et al. 1999). Similarly to the effect of exogenously applied noradrenaline, which decreases both IBAT SODs activity, the activity of these enzymes in the IBAT of rats acutely exposed to cold is also decreased.

From our present results it is also clear that noradrenaline decreases lipid peroxide production in the IBAT only. This is in agreement with the findings of Andorn and Pappolla (2001) and Sergeev et al. (1975) who found that some catecholamines (including noradrenaline) inhibit lipid peroxidation and have antioxidant action. This noradrenaline antioxidant action was associated with the presence of a free phenol group in their molecule. The mechanism of inhibition is the interaction of catecholamines with the free radicals leading to antioxidative protection. In this way noradrenaline may regulate the permeability of biological membranes. Bearing in mind the above mentioned data, we can conclude that noradrenaline induced the inhibition of the IBAT antioxidant enzymes (CuZnSOD, MnSOD, CAT) activities and the decrease in lipid peroxide concentration, which is probably mediated by NO i.e. by peroxynitrite. 
Unlike the IBAT, exogenous noradrenaline markedly increased catalase activity in the heart (Fig. 2), but had no effect on any of SODs activities studied. Similarly to the IBAT, L-NAME applied alone did not influence the activity of any of the three antioxidant enzymes in the heart. This NO synthase inhibitor did not prevent the noradrenaline-induced increase of catalase activity in the heart and was also without effect on the activity of any SODs. Based upon these results, it might be supposed that noradrenaline treatment probably increased the hydrogen peroxide production in the heart and consequently catalase activity, which is $\mathrm{H}_{2} \mathrm{O}_{2}^{-}$ scavenging enzyme. These results are in agreement with those of Brown et al. (1997), who have demonstrated that catalase activity in the heart can be increased by extracellular stimuli such as catecholamines. Since noradrenaline-induced increase of catalase activity in the heart was not abolished by L-NAME, it seems that mechanisms other than NO may be involved in its regulation.

In contrast to the IBAT, where exogenous noradrenaline decreases lipid peroxides production, this neurohormone was without effect in the heart. L-NAME also did not change the extent of lipid peroxidative processes. Thus, it is possible to assume that acutely applied noradrenaline did not influence NO production in the heart as shown by Kan et al. (1999) in the isolated rat cardiac myocytes.

In conclusion, these results indicate that both NA and L-NAME effects on antioxidant enzymes activity and lipid peroxide concentration are tissue-specific and suggest that nitric oxide mediates the noradrenalineinduced inhibition of antioxidant enzymes activity and lipid peroxidation in the interscapular brown adipose tissue only.

\section{Conflict of Interest}

There is no conflict of interest.

\section{Acknowledgements}

This study is supported by The Serbian Ministry of Science and Environmental Protection, Grant No 143050.

\section{References}

ANDORN AC, PAPPOLLA MA: Catecholamines inhibit lipid peroxidation in young, aged and Alzheimer's disease. Free Radic Biol Med 31: 15-320, 2001.

BEUTLER E Catalase. In: Red Cell Metabolism, a Manual of Biochemical Methods. E. BEUTLER (ed), Grune and Stratton, New York, 1982, pp 105-106.

BROWN GC: Regulation of mitochondrial respiration by nitric oxide inhibition of cytochrome c oxidase. Biochem Biophys Acta 1504: 46-57, 2001.

BROWN JM, GROSSO MA, TERADA LS, WHITMAN GJ, BANERJEE A, WHITE CW, HARKEN AH, REPINE JE: Endotoxin pretreatment increases endogenous myocardial catalase activity and decreases ischemiareperfusion injury of isolated rat hearts. Proc Natl Acad Sci USA 86: 2516-2520,1997.

CASSINA A, RADI R: Differential inhibitory action of nitric oxide and peroxynitrite on mitochondrial electron transport. Arch Biochem Biophys 328: 309-316,1996.

DAVIDOVIC V, DJOKIC I, PETROVIC N, DJURASEVIC S, CVIJIC G: Activity of antioxidant enzymes in rat skeletal muscle and brown fat: effect of cold and propranolol. J Thermal Biol 24: 385-389, 1999.

FINKEL MS, ODDIS CV, JACOB TD, WATKINS SL, HATTLER BG, SIMMONS RL: Negative inotropic effects of cytokines on the heart mediated by nitric oxide. Science 257: 387-389,1992.

GIORDANO A, TONELLO C, BULBARELLI A, COZZI V, CINTI S, CARRUBA OM, NISOLI E: Evidence for a functional nitric oxide synthase system in brown adipocyte nucleus. FEBS Lett 514: 135-140, 2002.

HIMMS-HAGEN J: Brown adipose tissue thermogenesis: interdisciplinary studies. FASEB J 4: 2890-2898, 1990.

KAN H, XIE Z, FINKEL MS: Norepinephrine-stimulated MAP kinase activity enhances cytokine-induced NO production by rat cardiac myocytes. Am J Physiol 276: H47-H52, 1999.

KANAI AJ, MESAROS S, FINKEL MS, CODDIS CV, BIRDER LA, MALINSKI T: B-Adrenergic regulation of constitutive nitric oxide synthase in cardiac myocytes. Am J Physiol 273: C1371-C1377, 1997.

KIKUCHI-UTSUMI K, GAO B, OHINATA H, HASHIMOTO M, YAMAMOTO N, KUROSHIMA A: Enhanced gene expression of endothelial nitric oxide synthase in brown adipose tissue during cold exposure. $\mathrm{Am} J$ Physiol 282: R623-R626, 2002. 
KOIVISTO A, MATTHIAS A, BRONNIKOV G, NEDERGAARD J: Kinetics of the inhibition of mitochondrial respiration by NO. FEBS Lett 417: 75-80, 1997.

KOSTIĆ TS, ANDRIĆ SA, MARIĆ D, KOVAČEVIĆ RZ: Inhibitory effects of stress-activated nitric oxide on antioxidant enzymes and testicular steroidogenesis. J Steroid Biochem Mol Biol 75: 299-306, 2000.

KREMSER K, STANGL H, PAHAN K, SINHG I: Nitric oxide regulates peroxisomal enzyme activities. Eur J Clin Chem Clin Biochem 33: 763-774, 1995.

MISRA HP, FRIDOVICH I: The role of superoxide anion in the autooxidation of epinephrine and a simple assay for superoxide dismutase. $J$ Biol Chem 247: 3170-3175, 1972.

NAGASHIMA T, OHINATA H, KUROSHIMA A: Involvement of nitric oxide in noradrenaline-induced increase in blood flow through brown adipose tissue. Life Sci 54: 17-25, 1994.

NAPOLI C, IGNARRO LJ: Nitric oxide and atherosclerosis. Nitric Oxide 5: 88-97, 2001.

NISOLI E, TONELLO C, BRISCINI L, CARRUBA MO: Inducible nitric oxide synthase in rat brown adipocytes: implications for blood flow to brown adipose tissue. Endocrinology 138: 676-682, 1997.

REHNCRONA SA, SMITH DS, AKESSON B, WESTERBERG E, SISEJO BK: Peroxidative changes in brain cortical fatty acids and phosfolipids as characterized during $\mathrm{Fe}^{2+}$ and ascorbic acid-stimulated lipid peroxidation in vitro. J Neurochem 34: 1630-1638, 1980.

RICQUIER D, CASSARD-DOULCIER AM: The biochemistry of white and brown adipocytes analyzed from a selection of proteins. Eur J Biochem 218: 785-796, 1993.

SAHA SK, KUROSHIMA A: Nitric oxide and thermogenic function of brown adipose tissue in rats. Jpn J Physiol 50: 337-342, 2000.

SERGEEV PV, SEIFULLA RD, DUNAEV VG, RUDNEV IN: Effect of adrenaline, noradrenaline, dopamine, DOPA and phenylalanine on lipid peroxidation in liver mitochondria membranes. Bull Exp Biol Med 80: 25-28, 1975.

TAKADA Y, NOGUCHI T, OKABE T, KAYIYOMA M: Superoxide dismutase in various tissues from rabbits bearing the Vx-2 carcinoma in the maxillary sinus. Cancer Res 42: 4233- 4235, 1982.

YAMAKURA F, TAKA H, FUJIMURA T, MURAYAMA K: Inactivation of human manganese-superoxide dismutase by peroxynitrite is caused by exclusive nitration of tyrosine 34 to 3-nitrotyrosine. J Biol Chem 273: 1408514089, 1998. 